\title{
\begin{tabular}{l|l} 
pcori). & PATIENT-CENTERED OUTCOMES RESEARCH INSTITUTE \\
RESEARCH SUMMARY
\end{tabular}
}

PROJECT INFORMATION

September 2018

\section{Developing Methods to Predict Health Outcomes in Trauma Patients}

Principal investigator

Alan Hubbard, PhD
Organization

University of California, Berkeley

\section{What was the research about?}

Electronic health records store a lot of data about a patient. These data often include race, age, health problems, current medicines, and lab results. Looking at these data may help doctors treating patients after a trauma predict how likely it is that they will respond well to a treatment and survive. This information can help doctors make better treatment decisions. But first, researchers need to figure out how to combine and analyze data to make accurate predictions.

In this study, the research team created new statistical methods to combine data from patient records. They used these methods to predict patient health outcomes. Then the team used health record data collected from patients in hospital trauma centers to test their predictions.

\section{What were the results?}

The research team made three new computer programs. The first program combined data from patient records to automatically predict patient outcomes. Using test data, the program predicted how likely trauma patients were to stop bleeding and to survive.

The second program looked at whether the new method could be used to show what information was likely to be important for predicting outcomes. For example, in a test case, head injury and blood clotting problems were linked with lower chances of survival among trauma patients.

The third program combined data from patients' records to automatically suggest a treatment choice. In a test case, the team looked at what would happen if patients received the treatments that the program chose. Using the treatments recommended by the program didn't lead to better patient outcomes.

\section{What did the research team do?}

The research team used math equations to combine data from trauma patients. The data included gender, age, weight, blood pressure, medicines, trauma severity, trauma site, blood clotting, and lab readings. Then the team used the equations to create the computer programs.

Next, the team tested the programs using data created to mimic real data. Then they tested the programs with data from medical records of patients with severe trauma.

\section{What were the limits of the study?}

The data used to create the computer programs weren't always complete. Missing information may have made the predictions less accurate. Also, the data didn't include information taken over time, such as hourly blood pressure readings. If the programs had included these data, they might have predicted different results.

Future research could look at adding other types of data to the programs. Studies could also test the computer programs with new patients who have had severe trauma or with patients with other health problems. 
How can people use the results?

Researchers could continue to test and improve the computer programs. The programs may eventually help doctors make treatment decisions based on patients' traits and other health problems.

To learn more about this project, visit www.pcori.org/Hubbard143. 